\title{
Variations in the Circumplex Structure of Mood
}

\author{
Lisa A. Feldman \\ Pennsylvania State University
}

Typically, researchers have emphasized the similarity of the semantic and self report mood circumplexes. The purpose of the present study was to investigate systematic differences in these structures. The semantic circumplex is defined by two dimensions: valence and level of arousal. The present study demonstrated that when making judgments of their mood, people weigh the arousal dimension less than the valence dimension, whereas in the semantic structure the two mood dimensions are weighed equally. This reduction in the size of the arousal dimension was directly associated with increases in the correlation between self reported anxiety and depression. The results are discussed with reference to the meaning of subjective mood ratings.

\section{Man} well represented as a circumplex (Larsen \& Diener, 1992; Russell, 1980; Watson 8c Tellegen, 1985). The circular structure of affect has been extracted from people's judgments of the similarity between pairs of mood terms (Block, 1957; Bush, 1973; Russell, 1978, 1980, 1983; Russell, Lewicka, 8c Niit, 1989; Russell \& Ridgeway, 1983), from perceptions of facially expressed emotion (e.g., Abelson $8 c$ Sermat, 1962; Cliff $8 c$ Young, 1968; Dittmann, 1972; Fillenbaum $8 c$ Rapaport, 1971; Green \& Cliff, 1975; Russell 8c Bullock, 1985; Russell et al., 1989; Schlosberg, 1952, 1954; Shepard, 1962), and from selfreports of moods (Almagor \& Ben-Porath, 1989; Meyer \& Shack, 1989; Russell, 1978,1980; Russell \&Mehrabian, 1977; Russell $8 c$ Steiger, 1982; Watson, Clark, $8 c$ Tellegen, 1984; Watson $8 c$ Tellegen, 1985; Zevon $8 c$ Tellegen, 1982). The circumplex has been obtained for different rating forms, time frames, mood items, and factor analytic procedures (Watson, 1988) and for both children and adults (Russell $8 c$ Ridgeway, 1983), and it may constitute a cross-cultural universal in the structure of mood (Almagor 8c Ben-Porath, 1989; Russell, 1983, 1991; Russell et al., 1989; Watson et al., 1984).

The mood circumplex is defined as a circular arrangement of terms around two dimensions. Various interpre- tations of the circumplex finding exist. The original two-dimensional scheme, which I will call the valencel arousal model, is defined by two bipolar dimensions, valence and level of arousal (Russell, 1980; Schlosberg, 1952). The valence dimension captures the hedonic quality of a mood experience. The arousal dimension refers primarily to the perception of arousal rather than the level of physiological activation (Russell, 1989). The valence/arousal circumplex has been found in semantic similarity data, perceptions of facial expression, and self-report data. A schematic representation of the valence/arousal model is presented in Figure 1.

Other interpretations of the mood circumplex have provided a somewhat different slant on the data. Some researchers have labeled the dimensions differently (Diener, Larsen, Levine, $8 c$ Emmons, 1985), translated the structure as two dimensions of arousal (Thayer, 1989), or offered a rotational variant of the valence/ arousal circumplex. One model, the negative affect/ positive affect model, is a rotational variant of valence/arousal structure, defining the circumplex with dimensions at a $45^{\circ}$ rotation to the arousal and valence dimensions (Watson $8 c$ Tellegen, 1985; Zevon $8 c$ Tellegen, 1982). Negative affect (NA) is defined as a unipolar general factor of subjective distress. Positive affect (PA) is defined as a unipolar dimension of pleasurable engagement with the environment, or the extent to which a person avows a "zest for life." This rotational scheme exists only in analyses of self-reported mood and

Author's Note: Portions of this research were conducted as part of my Author's Note: Portions of this research were conducted as part of my
doctoral dissertation and were supported in part by a Social Sciences and Humanities Research Council of Canada (SSHRCC) doctoral fellowship. I thank Michael Ross and Erik Woody for their guidance on this project. In addition. I thank Robert Sinclair and Gerry Sande for their help in collecting some of the data reported in this study. Correspondence should be addressed to Lisa Feldman Barrett, Department of Psychology, Pennsylvania State University, University Park, PA 16802.

PSPB, Vol. 21 No. 8, August 1995 806-817

(C) 1995 by the Society for Personality and Social Psychology, Inc. 


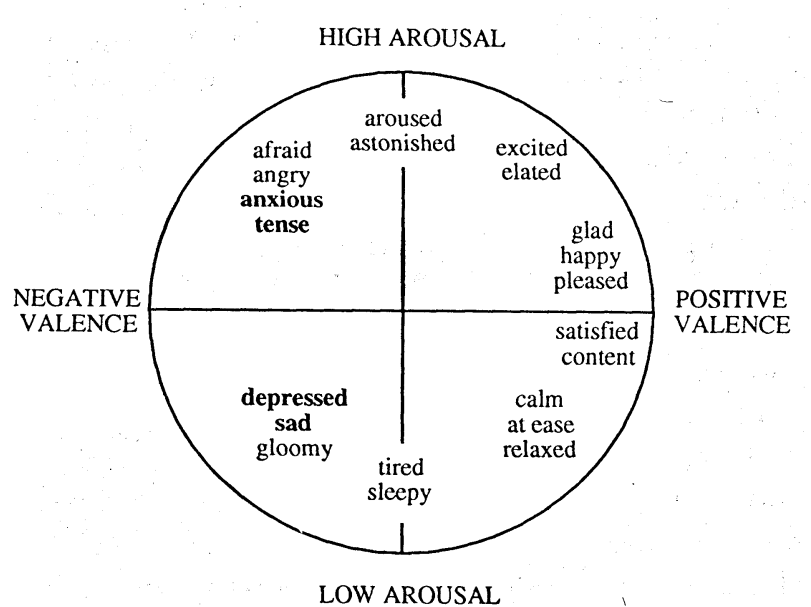

Figure 1 Valence/arousal circumplex model of mood adjectives. Based on Figures 2 and 3 from Russell (1980). Adapted by permission.

has not been the focus in analyses of similarity data or in perceptions of facial expressions. My hypothesis is built on the valence/arousal variation of the mood circumplex.

REDUCED IMPORTANCE OF

THE AROUSAL DIMENSION

Typically, researchers have emphasized the similarity among the circular structures obtained from similarity data, perceptions of facial expressions, and self-report ratings of mood (e.g., Russell, 1980, 1989; Watson \& Tellegen, 1985). Indeed, researchers have typically considered the similarities among the structures as evidence that people's semantic concepts influence the words that they choose to label and report their subjective experiences (Russell, 1980, 1989; Watson \& Tellegen, 1985; Zevon $8 c$ Tellegen, 1982). If one examines the various structures, however, there appear to be more differences than originally assumed. In particular, dimensional analyses of semantic similarity ratings and perceptions of facial expressions typically yield ideal circular structures (e.g., Russell, 1980, p. 1168; Russell et al., 1989). In contrast, factor analyses of self-reported mood typically yield elliptical structures (e.g., Russell, 1980, p. 1174), with a reduction in the size of the arousal dimension. This observation is consistent with factor analyses of self-report mood ratings in which the first unrotated factor, representing the valence of the mood ratings, typically accounts for twice as much variance as the second factor, representing arousal (e.g., Mayer \& Gaschke, 1988; Meyer \& Shack, 1989; Watson, 1988; Watson \& Tellegen, 1985). Though well known and noncontroversial, the observation that the arousal factor is substantially smaller in self-reported mood may be meaningful for understanding the meaning of the co- variations in emotion ratings. Researchers typically use a schematic representation of the self-report circumplex, however, rather than actual plots of the factor loadings, which leads to the false conclusion that all structures are identical.

The purpose of the present study is to investigate systematic differences in the semantic and self-report mood circumplexes. If semantic concepts are indeed associated with the way individuals interpret and communicate their affective experience, then- the semantic structure can be thought of as an ideal with an ideal circular shape. Its use in any particular domain (e.g., labeling faces, rating situations, rating one's own mood) may produce a structure that is influenced by the constraints of that domain. The observation that perceived arousal does not characterize self-reported mood to the degree that it is present in our semantic structure of mood adjectives may prove substantive in understanding the structure of affective experience and the nature of self-reported mood. When respondents use the semantic structure to label and report their mood experiences, they may weigh each dimension to arrive at a judgment. When subjects report their mood, they may reduce the extent to which they weigh the arousal dimension rather than weighing the two dimensions equally. To the extent that valence is a more dominant component of actual mood experiences, individuals may rate mood adjectives that are comparable in valence similarly. If I am feeling bad, I may use words such as upset, unhappy, or tense to describe my experience, even though the words imply different levels of arousal. If people are not attending to their level of arousal as much as to the valence of their affective experience, then the arousal-based similarity of mood adjectives may not be related to the way people rate their mood.

\section{DISCRIMINATING ANXIETY}

FROM DEPRESSION IN SELF-REPORT

One persistent problem in the measurement of emotions is the failure to discriminate anxiety from depression in self-report (Feldman, 1993). Despite an overwhelming theoretical consensus that anxiety and depression are different emotional experiences (e.g., Beck, 1976; Izard, 1977; Ortony, Clore, \& Collins, 1988; Pennebaker, 1982; Plutchik, 1980; Wallbott \& Scherer, 1989), researchers have generally been unable to separate them reliably. Whether psychologists have defined anxiety and depression as subjective mood states, traitlike personality entities, diagnostic or pharmacological entities, or behavioral or physiological patterns, the claim that depression and anxiety are discriminable constructs has not received consistent support (for reviews, see Clark, 1989; Clark 8c Watson, 1991; Dobson, 1985, 


\section{PERSONALITY AND SOCIAL PSYCHOLOGY BULLETIN}

Feldman, 1993; Maser \& Cloninger, 1990). Clear and consistent differentiation between measures of anxiety and depression is rarely achieved from self-reports of nonclinical, subclinical, or clinical samples (for a review, see Clark \& Watson, 1991; Feldman, 1993). Explanations implicating item overlap and response bias do not fully account for the discrepancy between theory and empirical evidence (see Feldman, 1990; Gotlib \& Cane, 1989; Tanaka-Matsumi \& Kameoka, 1986). Although respondents seem able to discriminate conceptually between anxiety and depression (e.g., Bush, 1973; Davitz, 1969; Pennebaker, 1982; Russell, 1980), they typically fail to do so when making judgments about their mood experience using inventorylike self-report measures. For example, the correlations between self-report measures of anxiety and depression typically exceed $r=.65$. Considering that the reliabilities of these scales typically range from $r=.49$ to $\mathrm{r}=.88$, with an average reliability of $r=.76$ (see Gotlib, 1984), such intercorrelations indicate that litde unique variance is being captured by anxiety and depression measures.

The notion that people weigh the arousal dimension less than the valence dimension when reporting their mood experiences may help explain why people fail to discriminate between anxiety and depression in selfreport. An examination of Figure 1 illustrates that anxiety and depression terms are similar in valence but different in the level of arousal they represent; anxiety terms denote a higher state of arousal than depression terms. These similarities and differences between anxiety and depression are consistent with people's intuitions (e.g., Davitz, 1969; Pennebaker, 1982; Russell $8 c$ Mehrabian, 1977; Wallbott $8 c$ Scherer, 1989) as well as with theoretical descriptions of the two moods (e.g., Pennebaker, 1982). The reduced size of the arousal dimension in the self-report circumplex suggests the possibility that when people report their mood, they may treat their level of arousal as a less significant part of their affective experience. The reduced weight of perceived arousal in self-report, then, may be a psychologically meaningful process that accounts for the failure to differentiate anxious mood from depressed mood.

\section{OVERVIEW OF THE PRESENT STUDY}

The goal of the present study was to test the hypothesis that people weigh the valence and arousal dimensions differently when reporting their mood states. I tested this proposal by asking one group of subjects for semantic similarity ratings of a group of mood terms and comparing these semantic ratings with self-reported mood ratings from three other samples of subjects. The data were analyzed in two ways to test my hypothesis. All ratings were first subjected to dimensional analyses. Consistent with previous findings, I predicted that the analysis of the semantic similarity ratings would yield a circumplex defined by valence and arousal dimensions. As in previous studies, I expected that two factors, valence and arousal, would account for a large proportion of the variance in the self-report ratings of mood, the valence factor accounting for more variance than the arousal factor. The smaller magnitude of the loadings of the mood words on the arousal factor should produce a two-dimensional elliptical solution rather than a perfect circle, reflecting the reduced role for arousal in the mood ratings. For the second set of analyses, I transformed the similarity ratings and the mood data to reflect the proximity of each word to every other word. I compared inter-item correlative of the mood with the valence-based and arousalbased similarity of the mood words to determine whether words that were similar in valence were rated comparably and whether words that were similar in level of arousal were rated comparably. I predicted that the pattern of subjects' mood ratings would be more strongly associated with the valence-based similarity of the mood terms than with the arousal-based similarity of the terms. Finally because the placement of words around the circumplex is associated with the correlations among those words (Russell, 1989; Watson $8 c$ Tellegen, 1985), I compared the proximity of anxiety and depression words in semantic and self-report two-dimensional space to determine whether the size of the arousal dimension was associated with the correlation between anxiety and depression ratings.

METHOD

\section{Subjects}

Semantic similarity ratings. A sample of five male and five female first-year undergraduate students from the University of Waterloo Psychology Department were asked to make judgments of semantic similarity for pairs of mood words. 1

Mood ratings. Three larger samples of subjects were asked for ratings of their current mood. The first sample consisted of 72 students registered in a University of Waterloo undergraduate course in statistics. The sample contained 58 women and 10 men; the sex of 4 subjects was unavailable. The second sample consisted of 120 first-year undergraduate students in the University of Alberta psychology department. The sample included 59 women and 51 men; the sex of 10 subjects was unknown. The third sample consisted of 177 male and 135 female first-year undergraduate students at the University of Manitoba. 


\section{Measures}

Semantic similarity measure. Sixteen terms were chosen from the affective circumplex. Two mood terms represented each pole of each dimension to ensure that all octants of the circumplex were equally represented (aroused, surprised, peppy, enthusiastic, happy, satisfied, calm, relaxed, quiet, still, sleepy, sluggish, sad, disappointed, nervous, afraid). The four anxiety-related terms (calm, relaxed, nervous, afraid) and the four depression-related terms (happy, satisfied, sad, disappointed) all appear in the Multiple Affect Adjective Check List subscales (MAACL; Zuckerman $8 c$ Lubin, 1965) used to assess self-reported anxious and depressed mood. Subjects rated the similarity of all possible pairs of mood terms (120). Similarity was rated on a 7 -point scale from 1 = extremely dissimilar, through $4=$ unrelated, to $7=$ extremely similar. Each mood term appeared as the first member in half of the pairs in which it occurred and as the second member in the other half, and the adjective pairs were presented in a single random order (Davison, 1983).

Mood measures. The mood questionnaire included the 16 mood terms chosen from the circumplex, as well as many other terms found in commonly used self-report measures of mood. Subjects indicated on a 7-point scale the extent to which each of 75 mood adjectives described their $\operatorname{mood}(0=$ not at all, $3=$ a moderate amount, $6=$ a great deal). Subjects were asked to rate how they felt right at that moment in time.

Several sets of commonly used anxiety and depression scales were constructed from the larger questionnaire. ${ }^{2}$ First, subjects' scores on the Emotions Questionnaire subscales (EQ Higgins, Klein, $8 c$ Strauman, 1985) were calculated. The EQ is a version of the MAACL designed to maximize the distinction between anxiety and depression. The terms that make up the EQ depression scale are energetic, happy, satisfied, proud, cheerful, enthusiastic, blue, discouraged, disappointed, ashamed, unworthy, and sad. The terms that make up the EQ anxiety scale are calm, secure, virtuous, peaceful, composed, relaxed, guilty, afraid, worried, nervous, anxious, and tense. Second, the Profile of Mood States anxiety and depression subscales (POMS; McNair, Lorr, \& Droppelman, 1971) were constructed. Third, the fear and sadness subscales of the Positive Affect and Negative Affect Schedule-Expanded Form (PANAS^X; Watson \& Clark, 1991) were also constructed.

\section{RESULTS}

The results are presented in three sections. First, I examine the semantic structure of mood and the structure of self-reported mood. Subsequently, I compare the inter-item correlations of the mood ratings obtained from the larger samples of subjects with the semantic distances of the mood words derived from the smaller sample. Finally, I examine the relationship between the size of the arousal dimension and the correlation between self-reports of anxious and depressed mood.

\section{Structures of Mood}

The semantic structure of mood. The similarity ratings from the sample of 10 respondents were subjected to a nonmetric group multidimensional scaling (MDS) analysis (Young, Takane, $8 c$ Lewyckyj, 1978) to provide a representation of the subjects' semantic structure of mood. Multidimensional scaling indicates the similarity between stimuli (in this case, mood adjectives) by their distance in a geometric space. The dimensions of the space are interpreted as the fundamental attributes that characterize the class of stimuli. The fit of a solution is determined by a stress value, which indicates how far the model departs from the observed data. A stress-bydimension plot is used to decide the appropriate number of dimensions for a solution; dimensions are added to the solution until the stress is low enough that it can no longer be significantly improved by the addition of more dimensions. A plot of the stress values by the number of dimensions for the MDS solution revealed a clear elbow at the two-dimensional solution, suggesting the suitability of that solution, Stress $=.18 .^{3}$ The mood terms fell, as predicted, in a circular order around two dimensions. Inspection of the arrangement of terms along each dimension suggested that one axis represented the level of arousal denoted by the mood terms and the other corresponded to the valence.

The structure of self reported mood. I examined the structure of self-reported mood using unrotated principal axis factor analyses. These analyses were designed to yield a structural representation of the dimensions underlying self-reported mood. Unrotated solutions yield valence and arousal dimensions, whereas solutions rotated to a varimax criterion yield underlying dimensions of negative affect and positive affect (Watson, 1988). If the semantic connections between mood terms are implicated in the rating process (Russell, 1980), then an inspection of the placement of mood terms in the structure of self-reported mood should reveal the way subjects use semantic structure to report their mood.

In the initial set of factor analyses, a three- or a four-factor solution was appropriate for all three samples of mood data. Both the scree test and the eigenvaluesgreater-than-one test were used as criteria for selecting the factor solution (Gorsuch, 1983). The four factor solutions were rotated, and the less robust factors (those with eigenvalues less than one) were dropped from the analyses. In the final solution, three factors were ex- 
810 PERSONALITY AND SOCIAL PSYCHOLOGY BULLETIN

TABLE 1: Factor Loadings From the Three Samples of Mood Ratings

\begin{tabular}{|c|c|c|c|c|c|c|c|c|c|}
\hline \multirow[b]{2}{*}{ Mood Term } & \multicolumn{3}{|c|}{ First Sample $(\mathrm{N}=72)$} & \multicolumn{3}{|c|}{ SecondSample $(\mathrm{N}=120)$} & \multicolumn{3}{|c|}{ Third Sample $(\mathrm{N}=312)$} \\
\hline & $F l$ & $F 2$ & $F 3$ & $F l$ & $F 2$ & $F 3$ & $F l$ & $F 2$ & $F 3$ \\
\hline Peppy & .713 & .485 & .335 & .596 & .377 & .253 & .725 & .456 & .147 \\
\hline Enthusiastic & .615 & .408 & .140 & .513 & .368 & .457 & .626 & .447 & .140 \\
\hline Happy & .785 & .040 & .242 & .806 & .001 & .245 & .783 & .222 & .219 \\
\hline Satisfied & .654 & .165 & .315 & .748 & -142 & .233 & .629 & .014 & .247 \\
\hline Calm & .496 & -.405 & .287 & .371 & -.616 & .311 & .411 & -.309 & .417 \\
\hline Relaxed & .458 & -.435 & .369 & .531 & -.435 & .053 & .637 & -.286 & .314 \\
\hline Quiet & -.016 & -.256 & .511 & -.138 & -.479 & .341 & -.058 & -.204 & .360 \\
\hline Still & -.130 & -.024 & .386 & -.171 & -.243 & .303 & -.026 & -.028 & .366 \\
\hline Sleepy & -.529 & -.324 & .352 & -.503 & -.451 & .389 & -.518 & -.267 & .482 \\
\hline Sluggish & -.579 & -.196 & .555 & -.501 & -.464 & .384 & -.621 & -.182 & .448 \\
\hline Sad & -.612 & .180 & .189 & -.578 & .064 & .029 & -.608 & .275 & .164 \\
\hline Disappointed & -.655 & .249 & .367 & -.753 & .169 & .264 & -.623 & .253 & .260 \\
\hline Afraid & -.456 & .420 & .072 & -.330 & .508 & .311 & -.448 & .493 & .083 \\
\hline Nervous & -.529 & .488 & .081 & -.549 & .264 & .251 & -.589 & .476 & .104 \\
\hline Aroused & -.268 & .383 & .255 & -.121 & .324 & .405 & -.042 & .396 & .223 \\
\hline Surprised & .429 & .465 & .125 & .230 & .436 & .349 & .285 & .333 & .123 \\
\hline
\end{tabular}

NOTE: $\mathrm{Fl}$ = first unrotated factor extracted in the solution; F2 = second unrotated factor extracted; F3 = third unrotated factor extracted.

tracted for all samples of mood data. In all analyses, the first two factors appeared to be valence and arousal dimensions, respectively. The factor loadings for each sample are presented in Table 1. Coefficients of congruence are presented in Table $2 .{ }^{4}$

The percentage of variance accounted for in each analysis is presented in Table 3 . As expected, the valence and arousal factors accounted for a substantial portion of the total variance in the relationships between the mood ratings. Further, comparisons of the percentages of common variance accounted for by each of the factors were close to those reported for previous factor analyses of mood ratings. In all three analyses, the valence factor accounted for more variance in the mood ratings than the arousal factor.

The valence and the arousal factor loadings of the mood terms are plotted in Figure 2, along with the MDS analysis of the semantic similarity ratings. As predicted, the plots of the factor loadings produced an elliptical, rather than a circular, ordering of terms around the dimensions. Furthermore, the standard deviations of the factor loadings from all three samples indicated that there was less variation in the item loadings on the arousal factor than on the valence factor $(s=.34$ vs. $s=.55$ for the first sample of mood ratings; $\mathrm{s}=.38 \mathrm{vs} . \mathrm{s}=.53$ for the second sample; $s=.30$ vs. $s=.55$ for the third sample).

The analyses presented thus far replicate previous findings in support of the hypothesis that subjects do not rely on arousal information when making judgments of their mood to the extent that arousal is represented in their semantic structure. These analyses were designed to yield a representation of the dimensions underlying self-reported mood, however, and they offer only indi-
TABLE 2: Coefficients of Congruence for the Factors From the Three Samples of Mood Ratings

Factor 1 (Valence) Factor 2 (Arousal) Factor 3

Sample 1 Sample 2 Sample 1 Sample 2 Sample 1 Sample 2

$\begin{array}{llll}\text { Sample } 2 & .98 & .91 & .83\end{array}$

$\begin{array}{lllllll}\text { Sample } 3 & .97 & .98 & .96 & .87 & .96 & .86\end{array}$

NOTE: When comparing factors across different sets of individuals, coefficients of congruence rather than factor scores should be computed (Gorsuch, 1983). All coefficients of congruence not listed were below .44 , with a mean of .08 .

rect evidence of the hypothesized relation between the semantic connections among the mood terms and the mood ratings. The next set of analyses was designed to examine this relationship more explicitly.

Semantic Similarity and

the Structure of Self-Reported Mood

I compared a series of similarity matrices to determine the degree to which the relationships among the mood ratings were accounted for by either valence-based or arousal-based semantic connections between mood terms. The similarity among the mood terms was quantified by their MDS distances from one another along each dimension. These distances were then compared with the covariation in the mood ratings. I predicted a strong correspondence between the covariation in ratings of the mood terms and the valence-based similarity of those terms and a smaller correspondence between the mood terms and arousal-based similarity.

As a measure of valence-based similarity, I calculated the absolute difference between the MDS valence di- 
TABLE 3: Portions of Variance Accounted for in the Factor Solutions of the Three Samples of Mood Ratings

\begin{tabular}{lclc}
\hline & & \multicolumn{2}{l}{$\begin{array}{l}\text { Percentage of Common } \\
\text { Variance Accounted For } \\
\text { by the First Two Factors }\end{array}$} \\
& $\begin{array}{c}\text { Percentage of Total Variance } \\
\text { Accounted For by the } \\
\text { First Two Factors }\end{array}$ & $\begin{array}{l}\text { Factor 1 } \\
\text { (Valence) }\end{array}$ & $\begin{array}{c}\text { Factor 2 } \\
\text { (Arousal) }\end{array}$ \\
\hline Sample 1 & $\mathbf{4 2 . 4}$ & $\mathbf{5 6}$ & $\mathbf{2 3}$ \\
Sample 2 & $\mathbf{4 0 . 2}$ & $\mathbf{5 3}$ & $\mathbf{2 8}$ \\
Sample 3 & 38.8 & $\mathbf{6 1}$ & $\mathbf{2 2}$ \\
\hline
\end{tabular}

mension coordinates for all pairs of mood terms; this produced 120 distances, one for each pair of mood terms. I calculated a measure of arousal-based similarity by assessing the distance between MDS arousal dimension coordinates for all pairs of terms. The smaller the distance between two terms on a dimension, the more similar those terms on the attribute represented by the dimension. I also created measures of similarity among the self-report mood ratings by correlating the ratings of each mood adjective with the ratings of every other mood adjective across subjects, to produce 120 correlations; these correlations were a measure of the similarity in ratings of the mood terms (Davison, 1983). Fisher r-to-z transformations were performed on these correlations so that they could be compared with the dimension distances. To produce an index of the degree to which words that are semantically similar are rated in the same way, I then correlated the attribute similarity data and the mood similarity data across the 120 pairs of mood terms. Correlations between similarity matrices were compared using a normal curve test for correlated correlation coefficients (Meng, Rosenthal, \& Rubin, 1992).

Comparisons of valence-based and arousal-based similarity with the similarity in mood ratings supported the hypothesis that words that are comparable in valence are rated more similarly than words that are comparable in the level of arousal they denote. The correlations are presented in Table 4. Valence-based and arousal-based similarity were both associated with correlations in the mood ratings. In all three samples, the valence-based similarity of the mood words was strongly associated with the mood ratings, whereas the arousal-based similarity of the words was less related to the mood ratings. ${ }^{5}$ Although the relationships were not statistically different in the first of the three samples, the valence-based similarity of the mood terms accounted for almost three times as much variance in this sample of mood ratings $(26 \%)$ as the arousal-based similarity of the words $(10 \%)$. Thus, there is support for the hypothesis that when making judgments about their mood experiences, subjects weigh valence information more heavily than arousal information. ${ }^{6}$
MDS Solution for the 16 Mood Terms

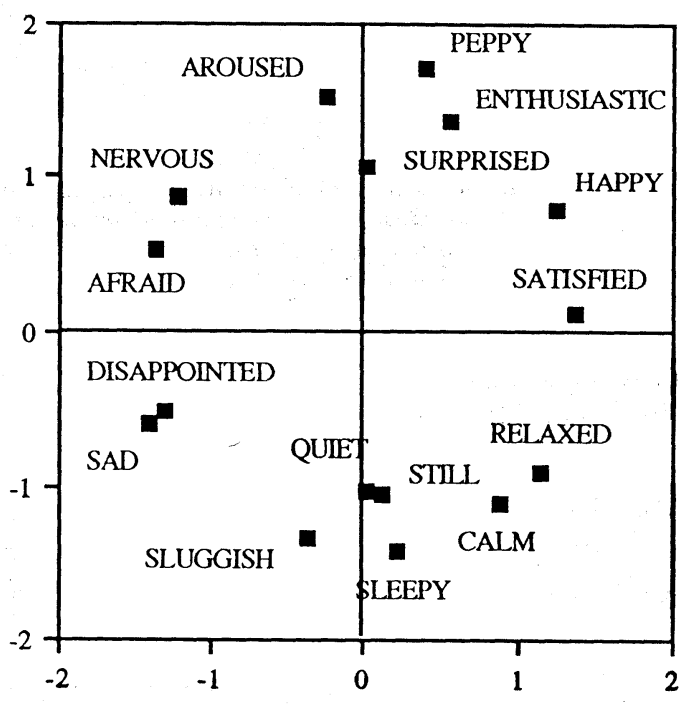

Factor Loadings for Mood

Ratings of 16 Mood Terms - Sample 1

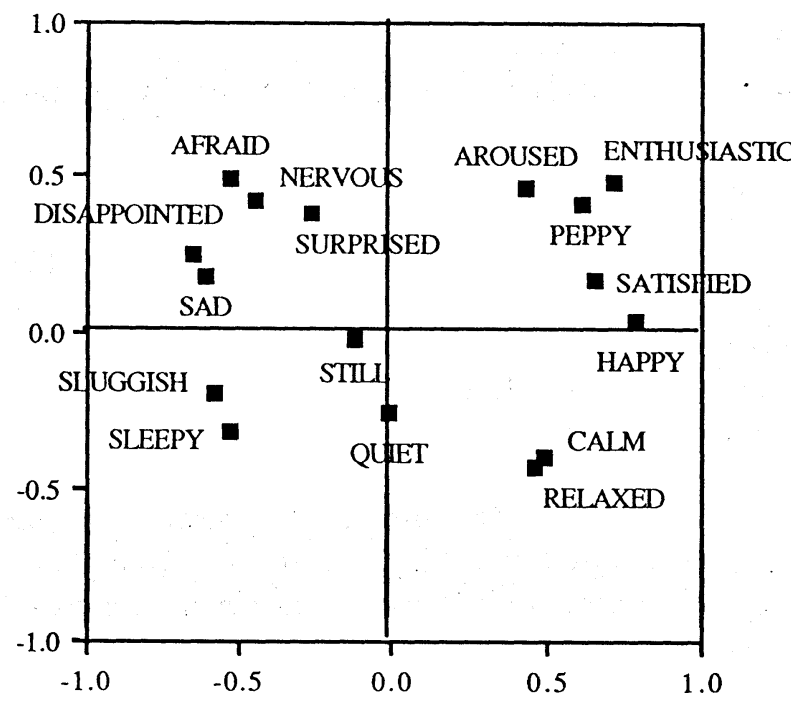

Figure 2 The MDS solution and the plot of factor loadings for the 16 mood adjectives. Valence is the horizontal axis and arousal $\checkmark \wedge \quad$ is the vertical axis for both plots.

\section{The Relationship Between}

Self-Reported Anxiety and Depression

In this section, I present evidence that the decreased importance of arousal information in self-report judgments is associated with the high correlation between 
TABLE 4: Zero-Order Correlations of Valence- and Arousal-Based Similarity With the Covariation in Self-Reported Mood

\begin{tabular}{lccc}
\hline Mood Ratings & $\begin{array}{c}\text { Arousal-Based } \\
\text { Similarity }\end{array}$ & $\begin{array}{c}\text { Valence-Based } \\
\text { Similarity }\end{array}$ & $\mathrm{z}$ \\
\hline First sample $(N=72)$ & .32 & .51 & $1.50^{*}$ \\
Second sample $(N=120)$ & .38 & .63 & $2.62^{\mathrm{b}}$ \\
Third sample $(N=312)$ & .30 & .60 & $5.08^{\mathrm{c}}$ \\
\hline
\end{tabular}

NOTE: The degrees of freedom for each correlation were based on the number of subjects involved in the calculation rather than on the number of mood pairs, because the pairs of mood terms were not independent from one another, and this procedure provided a more conservative estimate of degrees of freedom. The absolute values of the correlation coefficients are presented.

a.p $<.07$ (one-tailed)

b. $p<.005$ (one-tailed)

c.p<. 0001 (one-tailed)

anxious and depressed mood. In a circumplex structure, the correlation between any two terms is equal to the cosine of the angle between them (Russell, 1989; Watson $\&$ Tellegen, 1985). In the semantic structure presented in Figure 2, anxiety and depression terms are approximately $70^{\circ}$ apart, implying a correlation close to $r=.30$. For example, the words sad and nervous are $62^{\circ}$ apart, indicating a correlation between the two of $r=.47$; the words happy and relaxed are $70^{\circ}$ apart, reflecting a correlation of $r=.34$. Figure 3 shows what happens to the relationship between anxiety and depression terms when the semantic structure is modified such that the valence and arousal axes are of differential importance. The first structure in Figure 3 is the original semantic structure shown in Figure 2. Also presented are versions of what the semantic structure looks like with the size of the arousal dimension reduced by $25 \%, 50 \%$, and $75 \%$ from the original structure. By measuring the angles between anxiety and depression words in each of these structures, I was able to estimate the magnitude of the increase in the correlation between anxious and depressed mood ratings as the size of the arousal dimension decreased. These estimates are presented in Table 5. Also presented in Table 5 are the actual angles and associated correlations between ratings of anxious and depressed moods taken from the factor analyses of the three samples of mood ratings. Comparing the estimated and actual angles with their associated correlations, it appears that the size of the arousal dimension has decreased by between $50 \%$ and $75 \%$ from its size in the semantic structure.

The correlations between the self-report measures of anxious and depressed moods constructed from the data sets are somewhat lower, though generally consistent with the estimated correlations between individual items. The correlations hetween all anxious and depressed mood suhscales are presented in Table 6. The usual high correlations between depression and anxie subscales were found in all three samples. As can be see in Table 6, the EQ subscales were most highly correlate (average $r$ - .70), followed by the POMS subscales (ave age $r=.66$ ) and finally the PANAS-X subscales (averag $r=.50)$. The correlations corrected for attenuation du to unreliability seem to indicate that the size of th< arousal dimension in the self-report structure is betweei $25 \%$ and $50 \%$ smaller than in the semantic structure.

\section{DISCUSSION}

In summary, the findings of the present study suppor the hypothesis that when making judgments of theii mood, people weigh the arousal dimension less than the valence dimension, whereas in the semantic structure the two affect dimensions are weighed equally. The mood ratings did reflect perceived arousal to some de gree. Factor analyses of the mood data from all sample; demonstrated that an arousal factor did capture some o the variance in the mood ratings. Further, the correla tions between the arousal-based similarity of the moot words and the covariation in the actual mood rating: were significandy different from zero. Although self reported mood is not merely a judgment of valence these data support the conjecture that the structure ol self- reported mood does not conform to a true circumplex. This study demonstrated that the lack of circumplex structure in self-reported mood is not due, as some have suggested (e.g., Larsen \& Diener, 1992), to a failure to sample mood items from all the octants of the mood circumplex. It seems inaccurate, then, for researchers to continue to use schematic representations of the selfreport circumplex, rather than actual plots of the factor loadings (e.g., Meyer \& Shack, 1989), as this practice leads to the false conclusion that self-reported mood has a perfect circumplex structure.

The reduced importance of arousal in the structure of the self-report mood ratings as compared with size of the arousal dimension in the semantic structure was directly associated with an increased correlation between self-reported anxious and depressed moods. This observation indicates that the reduced importance of arousal in self-reported mood is psychologically meaningful and may perhaps help account for the failure of respondents to discriminate between like-valenced mood states in self-report.

\section{The Role of Perceived Arousal in Mood}

The results of this study clearly demonstrated that perceived arousal is an important component of the semantic connections among mood words and yet plays a more peripheral role in people's rating of their own mood. It is unclear why such a phenomenon wouldj 


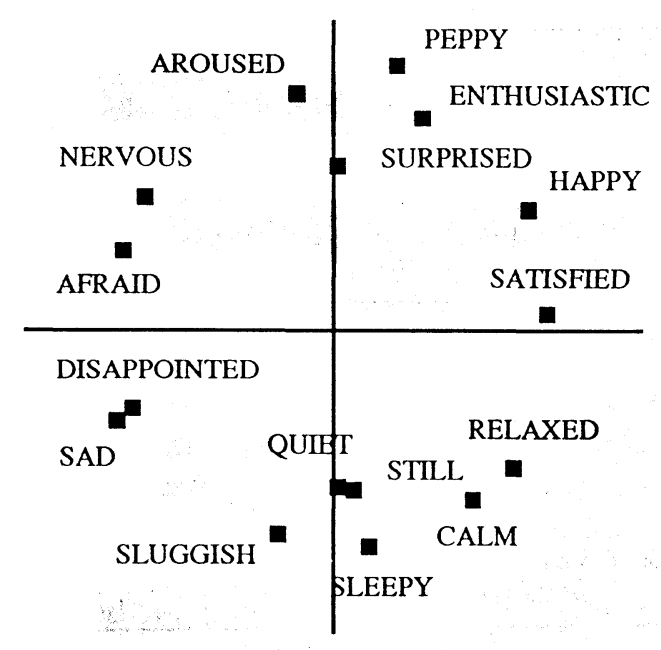

Original Semantic Circumplex

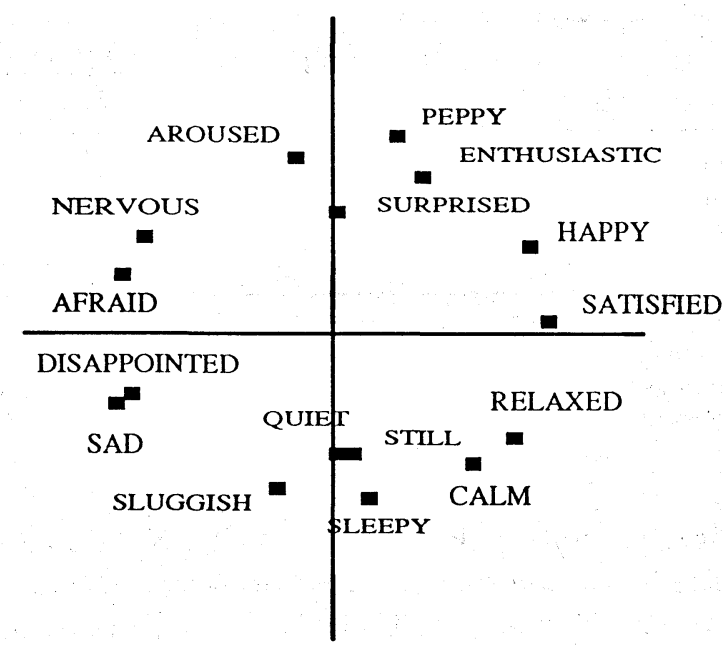

Semantic Circumplex with Arousal Dimension Reduced by $25 \%$

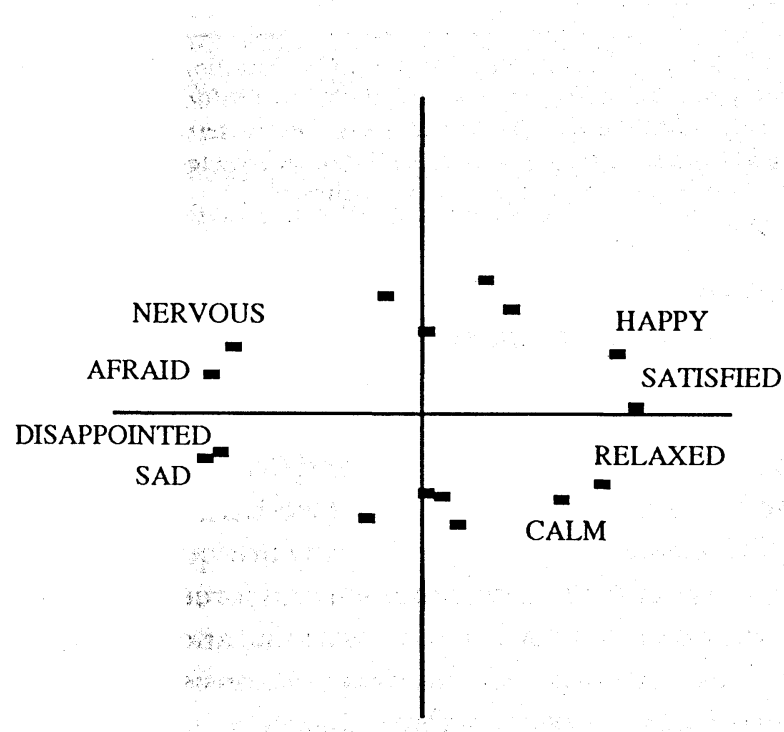

Semantic Circumplex with Arousal Dimension Reduced by $50 \%$

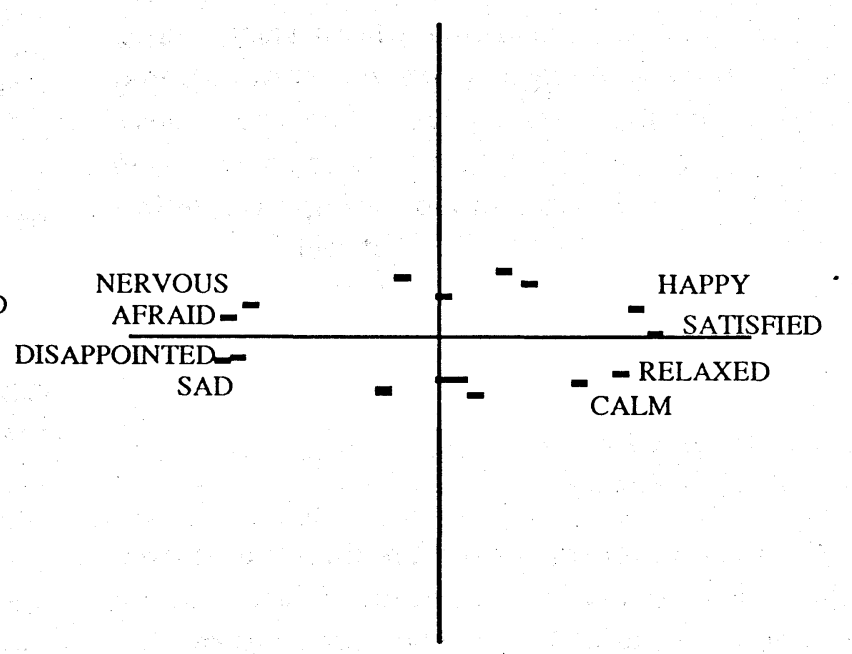

Semantic Circumplex with Arousal Dimension Reduced by $75 \%$

Figure 3 Changes in structural shape associated with reduced weight of the arousal dimension.

occur. The role of perceived arousal in the experience of mood remains unclear from a theoretical perspective. Some theorists consider perceived physiological arousal to be the immediate precursor of emotional labeling (e.g., Blascovich, 1992; Mandler, 1984; Schachter \& Singer, 1962); others suggest that the labeling of mood states primarily reflects evaluations of valence, with arousal noted afterward (Fiske, 1982; Pennebaker, 1982;
Weiner, 1982; Zajonc, 1980; for a discussion, see Fiske \& Taylor, 1991).

One theoretical possibility is that the importance of arousal information depends on the context in which mood ratings are made (Fiske, 1982; Pennebaker, 1982). Several researchers have suggested that most subjective moods may not be accompanied by arousal intense enough to orient people's attention to their internal 


\section{PERSONALITY AND SOCIAL PSYCHOLOGY BULLETIN}

TABLE 5: Estimated and Actual Angles With Associated Correlations

\begin{tabular}{|c|c|c|c|c|c|c|c|}
\hline & \multicolumn{4}{|c|}{ Semantic Circumplex } & \multicolumn{3}{|c|}{ Factor Analyses } \\
\hline & $0 \%$ & $25 \%$ & $50 \%$ & $75 \%$ & $S I$ & $S 2$ & $S 3$ \\
\hline Sad-nervous: angle & $62^{\circ}$ & $41^{\circ}$ & $33^{\circ}$ & $16^{\circ}$ & $42^{\circ}$ & $18^{\circ}$ & $15^{\circ}$ \\
\hline Sad-nervous: correlation & .47 & .75 & .84 & .96 & .74 & .95 & .97 \\
\hline Happy-relaxed: angle & $\mathbf{7 0}^{\circ}$ & $\mathbf{5 4}^{\circ}$ & $47^{\circ}$ & $20^{\circ}$ & $45^{\circ}$ & $38^{\circ}$ & $39^{\circ}$ \\
\hline Happy-relaxed: correllatiom & .34 & .59 & .68 & .94 & .71 & .79 & .78 \\
\hline
\end{tabular}

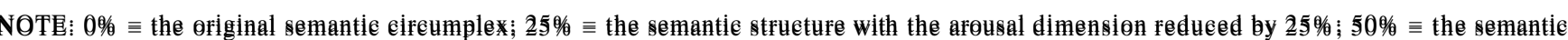
structure with the arousal dimension reduced by $50 \%$ \% 75 \% of mood fatings $(N \equiv 72)$; $\$ 2$ s seeond sample of mood fatings $(N \equiv 120)$; $\$ 3$ 三 thifd sample of mood fatings $(N \equiv 312)$ : The corfelations associated with the angles assume no measurement error:

physiological state (e.g., Pennebaker, 1982). Nor do subjects typically rate their mood in circumstances that are likely to orient them to their level of arousal. Thus, when neither internal nor external cues are strong enough to orient subjects to their physiological arousal, the weight of the arousal dimension may be reduced. This further suggests that the weight of the arousal dimension may vary in different contexts. Whereas the weight of arousal information may be determined contextually, the weight associated with valence may remain fairly stable across contexts. Presumably, in some cases, mood ratings may be influenced by perceived arousal, and the arousal dimension may account for more variance in mood ratings. As a consequence, mood ratings will reflect ratings of distinct mood states. Thus, depending on the weight of the arousal dimension in the mood judgment, self-report ratings may mean different things in different conditions.

\section{Anxiety and Depression as}

Distinct Psychological States

The findings support the notion that the arousal dimension influences judgments of mood less than the valence dimension, and this may be partly responsible for subjects' tendency not to differentiate anxiety from depression in self-report. The present findings also suggest that perceived arousal may have some role to play in understanding the precise relationship between anxiety and depression as clinical entities. The pattern of findings described for the self-report ratings has also been obtained in clinicians' ratings of patients (see D. A. Clark, Beck, \& Stewart, 1990; L. A. Clark, 1989; Stavrakaki $8 c$ Vargo, 1986). High correlations and singlefactor solutions typically characterize the research using clinically based ratings of anxious and depressed patients. A differentiation has been found only in correlational studies (e.g., Riskind, Beck, Brown, $8 c$ Steer, 1987 . Beck, Epstein, Brown, $8 c$ Steer, 1988) when clinicians' ratings are made in the context of the diagnostic process, with a mind to differential diagnosis between anxiety and
TABLE 6: Correlations Between Anxiety and Depression Subscales in Each Sample

Sample 1 (N-72 J Sample 2 Sample 3

$(N=312)$

$\begin{array}{llll}\text { EQ } & .69(.79) & .66(.75) & .74(.84) \\ \text { POMS } & .67(.76) & .63(.70) & .69(.77) \\ \text { PANAS-X } & .49(.54) & .43(.51) & .57(.64)\end{array}$

NOTE: Missing data deleted listwise. $\mathrm{EQ}=$ Emotions Questionnaire Anxiety and Depression subscales; POMS = Profile of Mood States Aubscales; PANAS-X = PANAS-X subscales. Internal consistencies 94. Correlations corrected for attenuation due to unreliability presented in parentheses.

depression (L. A. Clark, 1989). Clinicians likely adhere to the same theory as lay individuals: Anxiety and depression are distinguished by the level of arousal they denote. A plausible hypothesis, then, would be that the task of differential diagnosis is highlighted by the use of arousalbased information by clinicians (see Leff, 1978).

The conclusions from this study are qualified somewhat by several observations. First and foremost, the data presented in this study are correlational and do not verify any causal hypothesis. The data are consistent with the interpretation, however, that subjects reduce the extent to which they weigh the arousal dimension when reporting on their mood states. These data, though, do not confirm that subjects intentionally or unintentionally engage in this process to determine their own mood states.

Second, it has been argued that the range of mood terms sampled from the affective domain will determine the dimensions that can be extracted to define the structure of mood. Ortony, Clore, and their colleagues have suggested that arousal-related terms (e.g., aroused, sleepy, relaxed, sluggish, and surprised) are not true emotion words because they are not pure indicators of inner feeling states (Clore, Ortony, 8c Foss, 1987; Ortony, Clore, $8 c$ Foss, 1987). Such a claim challenges the validity of the valence/arousal structure as a model of emotional experience. They assert that inclusion of arousal-related 
terms ensures extraction of an arousal factor, even if the terms are not emotion words (Clore et al., 1987). The evidence in support of the Clore et al. hypothesis that arousal terms are not emotion words, however, is mixed (Storm \& Storm, 1987). Findings reported by Clore et al. (1987) provide only partial support for their claim that arousal-based words do not refer to true mood states. Subjects in their study rated words such as surprised and aroused as emotion words. Furthermore, people have trouble distinguishing among intense cognitive states, bodily sensations, and affective states. Terms that otherwise denote thoughts (e.g., relaxed, confused, stubborn) or physical sensations (e.g., jittery, weary, tired, sleepy, sluggish) seem more emotional when they become more intense (Clore et al., 1987). As Clore and his associates (1987) pointed out, the classification of emotion-related terms depends on the degree to which the affective aspect is focal to the meaning of the word, and in the end that is a subjective judgment. It seems unlikely, then, that the valence/arousal model of mood is an artifact of inappropriately including nonemotional words in the sample of mood terms. Moreover, the sample of mood words included in the present study cannot account for the observation that people weigh the valence and arousal dimensions equally when making judgments of semantic meaning but differentially when making judgments of their mood.

Finally, the present findings were not an artifact of restriction of range. The decreased size of the arousal dimension in the dimensional analyses of self-reported mood and the small correlations between the correlations in mood ratings and arousal-based similarity (Almagor \& Ben-Porath, 1989; Zevon $8 c$ Tellegen, 1982) do not appear to be caused by restriction of range in the ratings of arousal terms (Feldman, 1990). The range of ratings for arousal and valence terms was roughly comparable for all data sets. Indeed, the variances of the ratings of the arousal-related terms were as large as or larger than those of the valence-related terms, for the most part.

\section{Summary}

Self-reported mood does not conform to an ideal circumplex. The structure differs from the ideal circle of the semantic structure in that the size of the arousal dimension is decreased. This reduction in size of the arousal dimension was directly associated with the correlation between self-reported anxiety and depression and offers one perspective on why the two types of mood ratings are so highly correlated. Because semantic concepts are associated with the way individuals interpret and communicate their affective experience, use of the semantic structure for other types of mood ratings (e.g., labeling faces, rating situations) may produce a structure that is somewhat unique to those domains. A closer investigation of the variations in the circumplex structure in other types of mood ratings may improve our understanding of the many facets of affective experience.

\section{NOTES}

1. A sample of 10 subjects is adequate for a multidimensional scaling of 16 mood words where a two-dimensional solution is expected (Davison, 1983).

2. The word frightenedwas not included in the mood questionnaire Because of this oversight, the Fear scale of the PANAS-X was constructed without the item frightened. To minimize item overlap between anxiety and depression subscales, the items miserable, desperate, and terrified were not included in the POMS depression scale constructed in this study (Gotlib $8 c$ Cane, 1989).

3. Although Kruskal and Wish (1978) caution against accepting solutions with a Stress value above .10 , the elbow in the plot clearly appeared in the two-dimensional solution. I chose the two-dimensional solution on the basis of the plot and the relative interpretability of the various solutions (Davison, 1983).

4. The third factor in each solution was not readily interpretable a an acquiescent factor (Russell $8 c$ Steiger, 1982), a method factor, or a social desirability factor. The coefficients of congruence (Gorsuch, 1983) presented in Table 2 indicated that the unidentifiable factor wa all three analyses. Although other possible dimensions of mood have been identified in previous studies (e.g., Russell $8 c$ Mehrabian, 1977), they have not been consistently replicated across studies to the degree that the valence and arousal factors have. I did not pursue further the identification of the extra factor in any of the three analyses, given that it was not central to the theoretical hypothesis under investigation (Gorsuch, 1983).

5. These findings were replicated when the data from the three samples were combined to form two subsamples of mood ratings, one for men and one for women.

6. Item desirability was ruled out as a plausible explanation for the differential relationship of valence-based and arousal-based similarity to self-reported mood observed in the present study. Correlational analyses indicated that there was a strong association between the desirability of the mood words and the valence of the words, but the two were not identical entities. Valence- and desirability-based associations between the mood terms were uniquely related to the way the mood terms were rated (Feldman Barrett, 1995).

\section{REFERENCES}

Abelson, R. P., $8 c$ Sermat, V. (1962). Multidimensional scaling of facia expressions. Journal of Experimental Psychology, 63, 546-554.

Almagor, M., $8 c$ Ben-Porath, Y. S. (1989). The two-factor model of self-reported mood: A cross-cultural replication. Journal of Personality Assessment, 53, 10-21.

Beck, A. T. (1976). Cognitive theory and the emotional disorders. Madison, CT: International Universities Press.

Beck, A. T, Epstein, N., Brown, G., $8 c$ Steer, R. A. (1988). An inventory for measuring clinical anxiety: Psychometric properties. Journal of Consulting and Clinical Psychology, 56, 893-897.

Blascovich,J.J. (1992). A biopsychosocial approach to arousal regulation. Journal of Social and Clinical Psychology, 11, 213-237.

Block, J. (1957). Studies in the phenomenology of emotions. Journal of Abnormal and Social Psychology, 54, 358-363.

Bush, L. E. (1973). Individual differences multidimensional scaling of adjectives denoting feelings. Journal of Personality and Social Psychology, 25, 50-57.

Clark, D. A., Beck, A. T, $8 c$ Stewart, B. (1990). Cognitive specificity and positive-negative affectivity: Complementary or contradictory views on anxiety and depression? Journal of Abnormal Psychology, 99, 148-155. 


\section{PERSONALITY AND SOCIAL PSYCHOLOGY BULLETIN}

Clark, L. A. (1989). The anxiety and depressive disorders: Descriptive psychopathology and differential diagnosis. In P. C. Kendall $8 c$ D. Watson (Eds.), Anxiety and depression: Distinctive and overlapping features (pp. 83-129). Toronto: Academic Press.

Clark, L. A., $8 c$ Watson, D. (1991). Tripartite model of anxiety and depression: Psychometric evidence and taxonomic implications. fournal of Abnormal Psychology, 100, 316-336.

Cliff, N., $8 c$ Young, F. W. (1968). On the relation between unidimensional judgments and multidimensional scaling. Organizational Behavior and Human Performance, 3, 269-285.

Clore, G. L., Ortony, A., 8c Foss, M. A. (1987). The psychological foundations of the affective lexicon, fournal of Personality and Social Psychology, 53, 751-766.

Davison, M. L. (1983). Multidimensional scaling. Toronto: Wiley.

Davitz, J. R. (1969). The language of emotion. New York: Academic Press.

Diener, E., Larsen, R. J., Levine, S., $8 c$ Emmons, R. A. (1985). Intensity and frequency: Dimensions underlying positive and negative affect. Journal of Personality and Social Psychology, 48, 1253-1265.

Dittmann, A. T. (1972). Interpersonal messages of emotion. New York: Springer.

Dobson, K. S. (1985). An analysis of anxiety and depression scales. Journal of Personality Assessment, 49, 522-527.

Feldman, L. A. (1990). On the failure to differentiate anxiety and depression in self-report. Unpublished doctoral dissertation, University of Waterloo.

Feldman, L. A. (1993). Distinguishing depression and anxiety in selfreport: Evidence from confirmatory factor analysis on nonclinical and clinical samples. Journal ofConsulting and Clinical Psychology, 61 631-638.

Feldman Barrett, L (in press). Hedonic tone, perceived arousal, and item desirability: Three components of self-reported mood. Cognition and Emotion.

Fillenbaum, S., 8c Rapaport, A. (1971). Structures in the subjective lexicon New York: Academic Press.

Fiske, S. T. (1982). Schema-triggered affect: Applications to social perception. In M. S. Clark $8 c$ S. T. Fiske (Eds.), Affect and cognition: The seventeenth annual Carnegie Symposium on Cognition (pp. 55-78). Hillsdale, NJ: Lawrence Erlbaum.

Fiske, S. T., \& Taylor, S. E. (1991). Social cognition (2nd ed.). New York: McGraw-Hill.

Gorsuch, R. L. (1983). Factor analysis (2nd ed.). Hillsdale, NJ: Lawrence Erlbaum.

Godib, I. H. (1984). Depression and general psychopathology in university stud en ts. Journal of Abnormal Psychology, 93, 19-30.

Gotlib, I. H. 8c Cane, D. B. (1989). Self-report assessment of depression and anxiety. In P. C. Kendall $8 c$ D. Watson (Eds.), Anxiety and depression: Distinctive and overlapping features (pp. 131-169). Toronto: Academic Press.

Green, R. S., 8c Cliff, N. (1975). Multidimensional comparisons of structures of vocally and facially expressed emotion. Perception and Psychophysics, 17, 429-438.

Higgins, E.T., Klein, R., \&Strauman, T. (1985). Self-concept discrepancy theory: A psychological model for distinguishing among different aspects of anxiety and depression. Social Cognition, 3, 51-76.

Izard, C. E. (1977). Human emotions. New York: Plenum.

KruskalJ. B., $8 c$ Wish, M. (1978). Multidimensional scaling. Beverly Hills, CA: Sage

Larsen, R. J., $8 c$ Diener, E. (1992). Promises and problems with the circumplex model of emotion. Review of Personality and Social Psychology, 13, 25-59.

Leff, J. P. (1978). Psychiatrists' versus patients' concepts of unpleasant emotions. British Journal of Psychiatry, 133, 306-313.

Mandler, G. (1984). Mind and body: Psychology of emotion and stress. New York: Norton.

Maser, J. D., 8c Cloninger, C. R. (1990). Comorbidity of mood and anxiety disorders. Washington, DC: American Psychiatric Press.

Mayer, J. D., \& Gaschke, Y. N. (1988). The experience and metaexperience of mood. Journal of Personality and Social Psychology, 55 102-111.

McNair, D. M., Lorr, M., $8 c$ Droppelman, L. F. (1971). Manual for the Profile of Mood States. San Diego, CA: Educational and Industria Testing Service.
Meng, X., Rosenthal, R., \& Rubin, D. B. (1992). Comparing correlated correlation coefficients. Psychological Bulletin, 111, 172-175.

Meyer, G.J., $8 c$ Shack. J. R. (1989). Structural convergence of mood and personality: Evidence for old and new directions. Journal of Personality and Social Psychology, 57, 691-706.

Ortony, A., Clore, G. L., 8c Collins, A. (1988). The cognitive structure of emotions. New York: Cambridge University Press.

Ortony, A., Clore, G. L., $8 c$ Foss, M. A. (1987). The referential structure of the affective lexicon. Cognitive Science, 11,341-364.

Pennebaker, J. W. (1982). The psychology of physical symptoms. New York: Springer-Verlag.

Plutchik, R. (1980). Emotion: A psychoevolutionary synthesis. New York: Harper $8 c$ Row.

Riskind, J. H., Beck, A. T, Brown, G., $8 c$ Steer, R. A. (1987). Taking the measure of anxiety and depression: Validity of the reconstructed Hamilton scales. Journal oj Nervous and MentalDisease, 175, 474-479.

Russell, J. A. (1978). Evidence of convergent validity on the dimensions ofaffect. Journal of Personality and Social Psychology, 36, 1152-1168.

Russell, J. A. (1980). A circumplex model ofaffect. Journal of Personality and Social Psychology, 39, 1161-1178.

Russell, J. A. (1983). Pancultural aspects of the human conceptual organization of emotions. Journal of Personality and Social Psychology, $45,1281-1288$

Russell, J. A. (1989). Measures of emotion. In R. Plutchik $8 c$ H. Kellerman (Eds.),Emotion: Theory, research, andexperience (Vol. 4, pp. 83-111). Toronto: Academic Press.

Russell, J. A. (1991). Culture and the categorization of emotions. Psychological Bulletin, 110, 426-450.

Russell, J. A., $8 c$ Bullock, M. (1985). Multidimensional scaling of emotional facial expression: Similarity from preschoolers to adults. Journal of Personality and Social Psychology, 38, 1290-1298.

Russell, J. A., Lewicka, M., $8 c$ Niit, T. (1989). A cross-cultural study of a circumplex model of affect. Journal of Personality and Social Psychology, 57, 848-856.

Russell, J. A., $8 c$ Mehrabian, A. (1977). Evidence for a three-factor theory of emotions. Journal of Research in Personality, 11, 273-294.

RussellJ. A., $8 c$ Ridgeway, D. (1983). Dimensions underlying children's emotion concepts. Developmental Psychology, 19, 795-804.

RussellJ. A., \& Steiger,J. H. (1982). The structure in persons' implicit taxonomy ofemotions. Journal of Research in Personality, 16, 447-469.

Schachter, S., $8 c$ Singer, J. (1962). Cognitive, social, and physiological determinants of emotional states. Psychological Review, 69, 379-399.

Schlosberg, H. (1952). The description of facial expressions in terms of two dimensions. Journal of Experimental Psychology, 44, 229-237.

Schlosberg, H. (1954). Three dimensions of emotion. Psychological Review, 61,81-88.

Shepard, R. N. (1962). The analysis of proximities: Multidimensional scaling with an unknown distance function. Psychometrika, 27, 219246.

Stavrakaki, C, $8 c$ Vargo, B. (1986). The relationship of anxiety and depression: A review of the literature. British Journal of Psychiatry, 149, 7-16.

Storm, C, 8c Storm, T. (1987). A taxonomic study of the vocabulary of emotions. Journal ofPersonality and Social Psychology, 53, 805-816.

Tanaka-Matsumi, J., 8c Kameoka, V. A. (1986). Reliabilities and concurrent validities of popular self-report measures of depression, anxiety, and social desirability. Journal of Consulting and Clinical Psychology, 54, 328-333.

Thayer, R. E. (1989). The biopsychology of mood and arousal New York: Oxford University Press.

Wallbott, H. G., $8 c$ Scherer, K. R. (1989). Assessing emotion by questionnaire. In R. Plutchik $8 c$ H. Kellerman (Eds.), Emotion: Theory, research, and experience (Vol. 4, pp. 55-82). Toronto: Academic Press.

Watson, D. (1988). The vicissitudes of mood measurement: Effects of varying descriptors, time frames, and response formats on measurements of positive and negative affect. Journal of Personality and Social Psychology, 55, 128-141.

Watson, D., 8c Clark, L. A. (1991). Preliminary manual for the Positive Affect and Negative Affect Schedule (Expanded Form). Unpublished manuscript, Southern Methodist University.

Watson, D., Clark, L. A., $8 c$ Tellegen, A. (1984). Cross-cultural convergence in the structure of mood: A Japanese replication and a 
comparison with U.S. findings. Journal of Personality and Social Psychology, 47, 127-144.

Watson, D., $8 c$ Tellegen, A. (1985). Towards a consensual structure of mood. Psychological Bulletin, 98, 219-235.

Weiner, B. (1982). The emotional consequences of causal attributions. In M. S. Clark \& S. T. Fiske (Eds.), Affect and cognition: The seventeenth annual Carnegie Symposium on Cognition (pp. 185-209). Hillsdale, NJ: Lawrence Erlbaum.

Young, F. W., Takane, Y, \& Lewyckyj, R. (1978). ALSCAL: Anonmetric multidimensional scaling program with several different options. Behavioral Research Methods and Instrumentation, 10, 451-453.

Zajonc, R. B. (1980). Feeling and thinking: Preferences need no inferences. American Psychologist, 35, 151-175.
Zevon, M. A., $8 c$ Tellegen, A. (1982). The structure of mood change: An idiographic/nomothetic analysis. Journal ojPersonality and Social Psychology, 43, 111-122.

Zuckerman, M., \& Lubin, B. (1965). Manual for the Multiple Affect Adjective Check List. San Diego, CA: Educational and Industrial Testing Service.

Received July 6, 1993

Revision received February 23, 1994

Accepted February 23, 1994 